\title{
NOP14 regulates the growth, migration, and invasion of colorectal cancer cells by modulating the NRIP1/GSK-3 $\beta / \beta$-catenin signaling pathway
}

\author{
Xuanjin Zhu, ${ }^{1}$ Weilu Jia, ${ }^{2 *}$ Yong Yan, ${ }^{1}$ Yong Huang, ${ }^{1}$ Bailin Wang ${ }^{1}$ \\ ${ }^{1}$ Department of General Surgery, Guangzhou Red Cross Hospital, Medical College of Jinan University, Guangzhou \\ ${ }^{2}$ School of Clinical Medicine, Guizhou Medical University, Guiyang, China \\ * Present address: School of Medicine, Southeast University, Nanjing, China
}

\begin{abstract}
Colorectal cancer (CRC) is the third most common cancer diagnosed worldwide. Recently, nucleolar complex protein 14 (NOP14) has been discovered to play a critical role in cancer development and progression, but the mechanisms of action of NOP14 in colorectal cancer remain to be elucidated. In this study, we used collected colorectal cancer tissues and cultured colorectal cancer cell lines (SW480, HT29, HCT116, DLD1, Lovo), and measured the mRNA and protein expression levels of NOP14 in colorectal cancer cells using qPCR and Western blotting. GFP-NOP14 was constructed and siRNA fragments against NOP14 were synthesized to investigate the importance of NOP14 for the development of colorectal cells. Transwell migration assays were used to measure cell invasion and migration, CCK-8 kits were used to measure cell activity, and flow cytometry was applied to the observation of apoptosis. We found that both the mRNA and protein levels of NOP14 were significantly upregulated in CRC tissues and cell lines. Overexpression of GFP-NOP14 markedly promoted the growth, migration, and invasion of the CRC cells HT19 and SW480, while genetic knockdown of NOP14 inhibited these behaviors. Overexpression of NOP14 promoted the expression of NRIP1 and phosphorylated inactivation of GSK-3 $\beta$, leading to the upregulation of $\beta$-catenin. Genetic knockdown of NOP14 had the opposite effect on NRIP1/GSK-3/ $\beta$-catenin signals. NOP14 therefore appears to be overexpressed in clinical samples and cell lines of colorectal cancer, and promotes the proliferation, growth, and metastasis of colorectal cancer cells by modulating the NRIP1/GSK-3 $/ / \beta$-catenin signaling pathway.
\end{abstract}

Key words: Colorectal cancer; NOP14; proliferation; migration; invasion.

Correspondence: Bailing Wang, Department of General Surgery, Guangzhou Red Cross Hospital, Medical College of Jinan University, Guangzhou 510220, China. E-mail: bailinwang0314@163.com

Contribution: $\mathrm{XZ}$ and $\mathrm{WJ}$ contribute equally to this study. BW conceived the study and designed the experiments. XZ and WJ completed the experiment, analyzed the data and wrote the manuscript. YY and YH discussed the results. BW revised the manuscript.

Conflict of interest: The authors declare that they have no competing interests.

Availability of data and materials: The datasets used and/or analyzed during the current study are available from the corresponding author.

Ethical Approval: Ethical approval was obtained for all experimental procedures by the Ethical Committee of the Guangzhou Red Cross Hospital, Medical College of Jinan University.

Patient consent for publication: Written informed consent was obtained from the patients for their anonymized information to be published in this article. 


\section{Introduction}

Colorectal cancer (CRC) is a common digestive tract cancer, with the third-highest incidence of malignant cancers. The incidence of CRC is nearly 140 million people each year, leading to approximately 693,900 deaths. ${ }^{1,2}$ The worldwide prevalence of CRC is increasing year by year. ${ }^{3}$ Recently, with changes in diet, improvements in living standards, and worsening environmental conditions, the incidence of CRC in men has increased over those of lung and stomach cancer, and CRC has a higher incidence then breast and cervical cancer. ${ }^{4}$ Aging, obesity, alcohol consumption, smoking, low physical activity, and environmental factors are major risk factors for colon cancer. ${ }^{5-7}$ Radiotherapy, chemotherapy, and targeted therapy methods are used for the clinical treatment of CRC. ${ }^{8-10}$ Although the resection rate and cure rate of $\mathrm{CRC}$ has improved significantly in recent years, the five-years survival rate and overall cure rate of CRC have not noticeably increased. ${ }^{11-13}$ The prognosis of the diseases is poor, with a five-year survival rate of around 50\%. ${ }^{5}$ The major pathways which could be targeted for CRC therapy are epidermal growth factor receptor, ${ }^{14-16}$ VEGF/VEGFR, ${ }^{17,18}$ and the CDK8/ $\beta$-catenin, ${ }^{19}$ Hedgehog, ${ }^{20}$ Notch, ${ }^{21}$ TGF $\beta$-Smashs, ${ }^{22}$ Jak-STAT, ${ }^{23}$ Ras-Raf, and PI3K-Akt$\mathrm{mTOR}^{24}$ signaling pathways. However, the underlying mechanism of CRC is not fully understood.
Human nucleolar protein 14 (NOP14) is a gene located on chromosome $4 \mathrm{p} 16.317$. Studies have shown that NOP14 is involved in the precursors and processing of ribosomal RNA, and the synthesis of the mature $40 \mathrm{~s}$ ribosomal subunit. ${ }^{25}$ Kuhn et al. demonstrated that loss of expression of NOP14 leads to a reduction of the number of precursor $27 \mathrm{sA} 220$ s ribosomal RNAs, and upregulation of pre-23s and 35 s ribosomal RNA. ${ }^{26}$ NOP14 deletion may lead to Wolf-Hirachhorn (WH) syndrome. ${ }^{27}$ According to recent research, NOP14 participates in cancer development and progression. NOP14 inhibits the migration and invasion of breast cancer via the NRIP1/Wnt/ $\beta$ catenin pathway. ${ }^{28} \mathrm{miR}-502-5 \mathrm{p}$-regulated NOP14 suppresses bladder cancer development by regulation of cancer cell migration and proliferation. ${ }^{29}$ In addition, NOP14 is highly expressed in pancreatic cancer, and the overexpression of NOP14 promotes the invasion and migration of pancreatic cancer cells in vitro. ${ }^{30}$ NOP14, an oncogene, therefore plays a critical role in the growth, migration, and invasion of cancer cells.

In this study, we investigated the role of NOP14 in CRC. We found that NOP14 is upregulated in both CRC samples and cultured CRC cell lines. Overexpression of NOP14 promoted the proliferation, invasion, and migration of CRC cells while inhibition of NOP14 suppressed these behaviors.
A
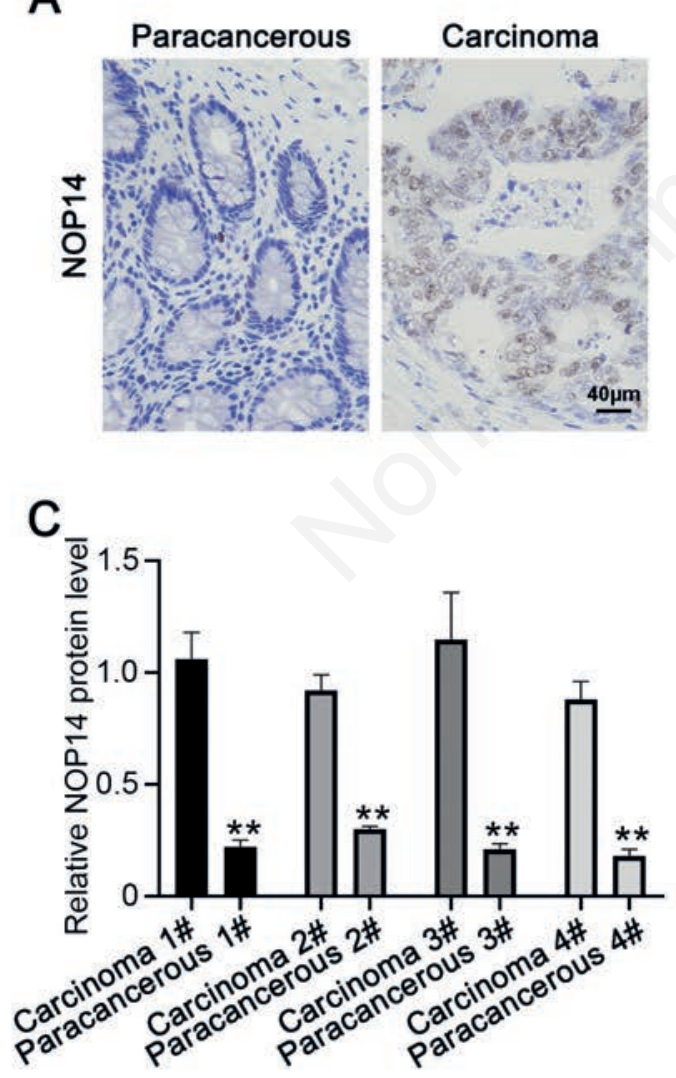

B

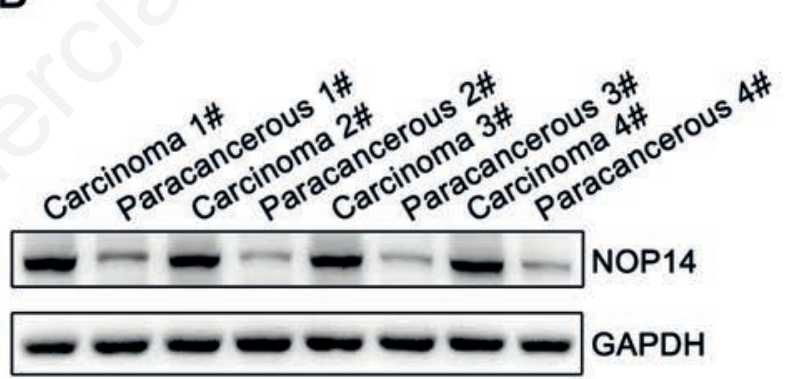

D

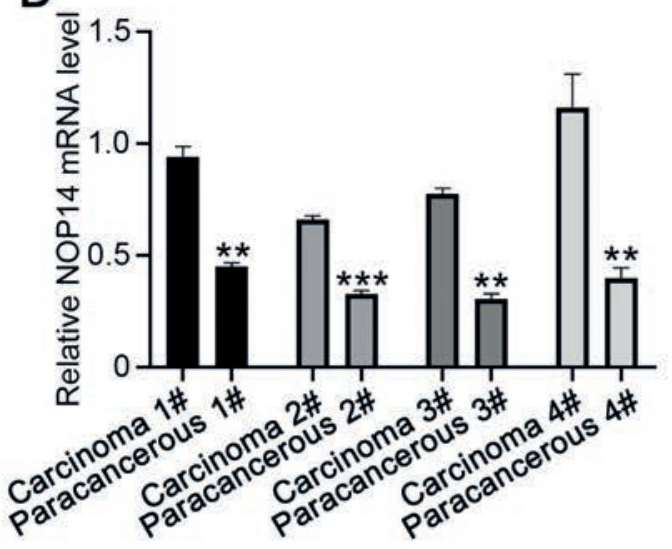

Figure 1. NOP14 upregulated in CRC. A) Immunohistochemical detection of benign CRC tissues and the expression levels of adjacent tissues of NOP14; scale bar, $50 \mu \mathrm{m}$. B-C) Western blotting detection of CRC clinical adjacent four cases of benign and cancer tissue correspond NOP14 protein. D) RT-PCR detection of four cases of clinical corresponding adjacent benign CRC, and cancer of mRNA expression NOP14. ${ }^{*} \mathrm{p}<0.05 ;{ }^{* *} \mathrm{p}<0.01$. 


\section{Materials and Methods}

\section{Tissue sampling}

All CRC tissue samples were handled following Standard Operating Procedures established by the study team at the start of the study ${ }^{31}$. Adjacent non-tumor tissues were also collected for comparison. The resected specimens were transported to the pathology department immediately following removal of the specimen from the patient. At the pathology department the specimens were handled at room temperature, and were snap frozen within two hours after resection. Written consent was obtained from all patients, and the study was approved by the ethics committee of the Guangzhou Red Cross Hospital, Medical College of Jinan University.

\section{Cell culture and transfection}

Human melanoma cell line 293T, a normal human colon mucosal epithelial cell line, NCM460, and the CRC cell lines HCT116, HT29, Lovo, SW480, and DLD1 were purchased from the Cell Bank of the Chinese Academy of Sciences (Shanghai, China). All cell lines were maintained in HyClone Dulbecco's modified Eagle's medium (DMEM) (Hyclone, Logan, UT, USA) containing 10\% fetal bovine serum (FBS) (Gibco, Thermo Fisher Scientific, Inc.) and $100 \mathrm{U} / \mathrm{mL}$ of penicillin and streptomycin (Thermo Fisher Scientific, Waltham, MA, USA) at $37^{\circ} \mathrm{C}$ in a humid atmosphere containing 5\% carbon dioxide. PCR amplification was used to obtain full-length human NOP14 cDNA, which was inserted into a pcDNA3.1 carrier according to the manufacturer's instructions (Realgene, Nanjing, China). Cells were seeded at a concentration of $1 \times 10^{5} /$ well in 24 -well plates. Using FuGENE HD transfection reagent (Roche Applied Science, Indianapolis, USA), according to the manufacturer's instructions, the NOP14 overexpression or inhibitor vectors were transfected into cells. The cells were maintained at $37^{\circ} \mathrm{C}$ under $5 \% \mathrm{CO}_{2}$. Forty-eight hours after transfection, the cells were harvested for quantitative reverse transcription-polymerase chain reaction (qRT-PCR) and Western blotting analyses.

\section{Western blotting}

Ice-cold radioimmunoprecipitation assay (RIPA) buffer (Beyotime, Shanghai, China) containing protease inhibitors (Invitrogen, California, USA) and methyl sulfonic acid benzyl ester (PMSF) (Invitrogen, Carlsbad, CA, USA) was used to disrupt the cells. The protein levels were quantified using dioctanic acid (BCA) protein assays (Thermo Fisher Scientific, MA, USA). Samples containing the same amount of protein were separated using sodium dodecyl sulfate-polyacrylamide gel electrophoresis, transferred to polyvinylidene fluoride membrane (Thermo Fisher Scientific), and incubated overnight with $10 \%$ skim milk at $4{ }^{\circ} \mathrm{C}$. After washing three times with phosphate buffer containing twain 20 (PBST) saline (PBS), the film was incubated for $1 \mathrm{~h}$ at room temperature with antibodies against NOP14 (1:500), phosphorylated GSK-3 $\beta$ (1:500), total GSK-3 $\beta$ (1:1000), $\beta$-catenin $(1: 1000)$, NRIP1 (1:1000), or GAPDH (1:2000), all of which were purchased from Abcam (Cambridge, MA, USA). After washing three times with PBST, the membrane was incubated with a 1:10000 dilution of horseradish peroxidase (HRP) conjugated goat anti rabbit IgG H\&L (Southern Biotech, Birmingham, AL, USA). The membrane was rinsed, and Enhanced Chemiluminescence Detection Kits (Thermo Fisher Scientific) were used to visualize the protein bands.

\section{CCK-8 assays}

HT29 and SW480 cells were grown to log phase, cell suspen- sions prepared at a density of $1 \times 10^{5}$ cells per $\mathrm{mL}$ and seeded into 96-well cell culture plates at $200 \mu \mathrm{L} /$ well under $37^{\circ} \mathrm{C}, 5 \% \mathrm{CO}_{2}$ Cell Counting Kit-8 (CCK8) solution (Thermo Fisher Scientific) was added at $10 \mu \mathrm{L} /$ well at $24 \mathrm{~h}, 48 \mathrm{~h}, 72 \mathrm{~h}, 96 \mathrm{~h}$ or $120 \mathrm{~h}$. After 2-4 h further culture, changes in the cells were observed, and the absorbance measure at $450 \mathrm{~nm}$ to evaluate the cell proliferation. The cell viability was calculated as viability $(\%)=[\mathrm{A}$ (treatment)A (blank) $] /[\mathrm{A}$ (no treatment)-A (blank) $] \times 100$, where $\mathrm{A}$ is the measured absorbance.

\section{Detection of apoptosis}

Annexin V-FITC/PI Dual Staining Kits (Biolegend, San Diego, CA, USA) was used for the assessment of cell apoptosis. Flow cytometry analysis was performed in accordance with the manufacturer's instructions (BD Biosciences, San Jose, CA,

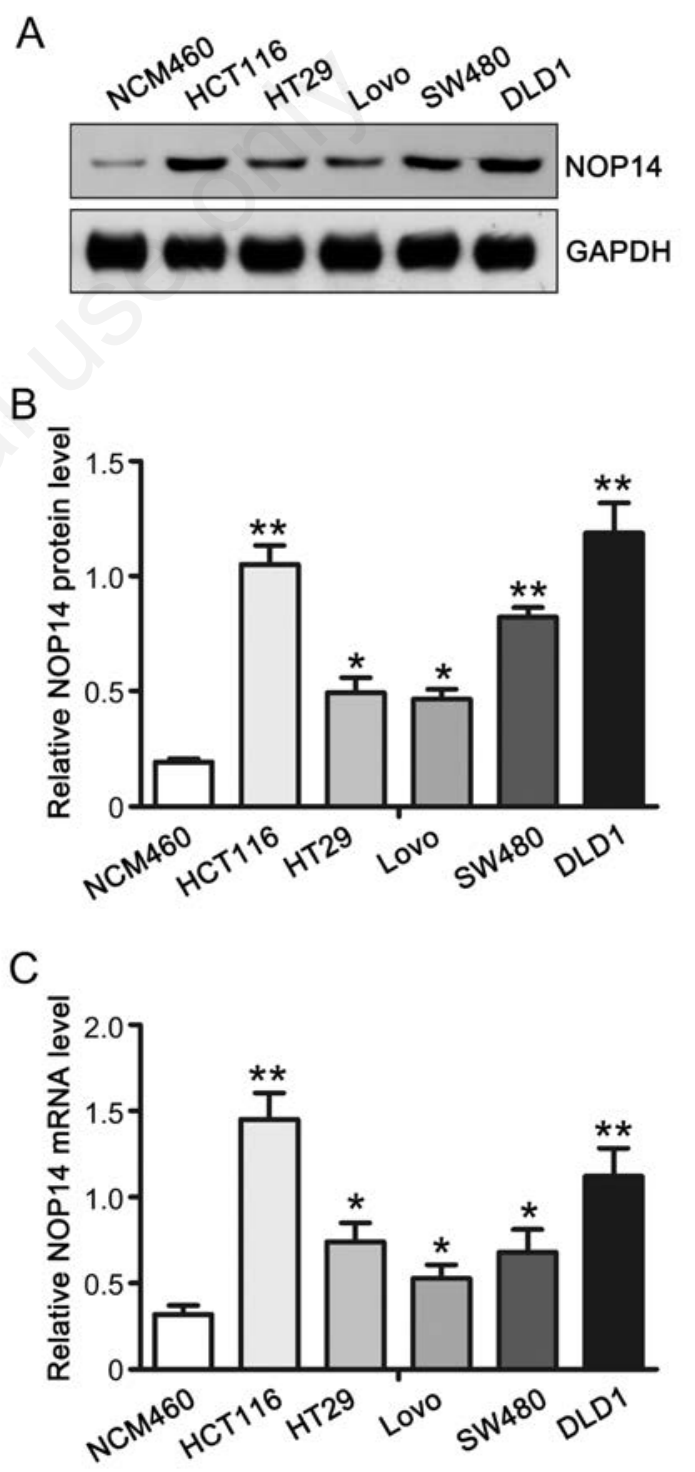

Figure 2. NOP14 upregulation of CRC cells. A) The expression of NOP14 was detected by Western blotting in a collection of normal colonic epithelial cells and five CRC cells. B) The quantified data in A was shown. C) RT-PCR detection of the mRNA expression of NOP14 in normal colon epithelium and colorectal cancer cells. . ${ }^{*} \mathbf{p}<0.05 ;{ }^{* *} \mathbf{p}<0.01$. 
USA). Cells were digested with trypsin without EDTA (Thermo Fisher Scientific) and collected, and the cells were washed twice with $4^{\circ} \mathrm{C}$ pre-chilled PBS. Then the binding buffer was diluted with deionized water at a ratio of 1:3, and the cells resuspended with binding buffer to adjust the concentration to $1 \times 10^{6}$ cells $/ \mathrm{mL}$. Each $100 \mu \mathrm{L}$ aliquot of cell suspension was placed into a flow tube and Annexin V/FITC and $10 \mu \mathrm{L}$ of $20 \mu \mathrm{g} / \mathrm{mL}$ PI solution were added. The solution was thoroughly mixed, and incubated at room temperature in the dark for $15 \mathrm{~min}$. Then, $400 \mu \mathrm{L}$ of PBS (Thermo Fisher Scientific) was added to the reaction tube, and early apoptosis analyzed using a flow cytometer. All experiments were repeated at least three times.

\section{Migration and invasion assays}

Cells were starved in serum-free medium for 8-12 h, and then trypsinized to prepare a cell suspension, and counted. The cell sus-
A

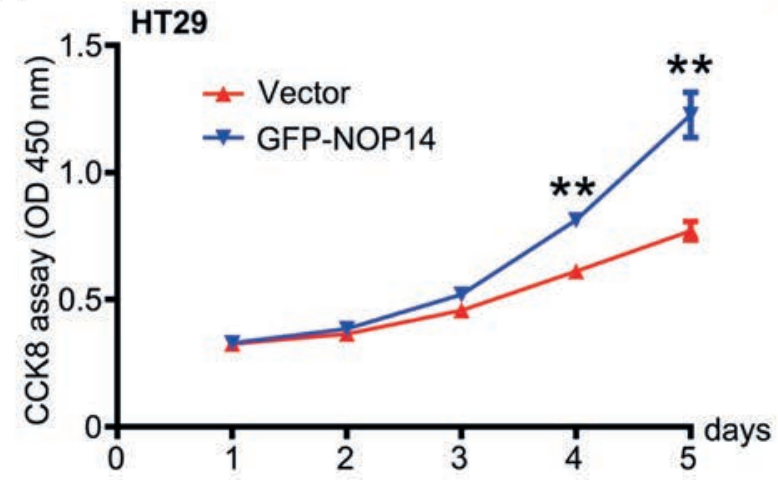

C

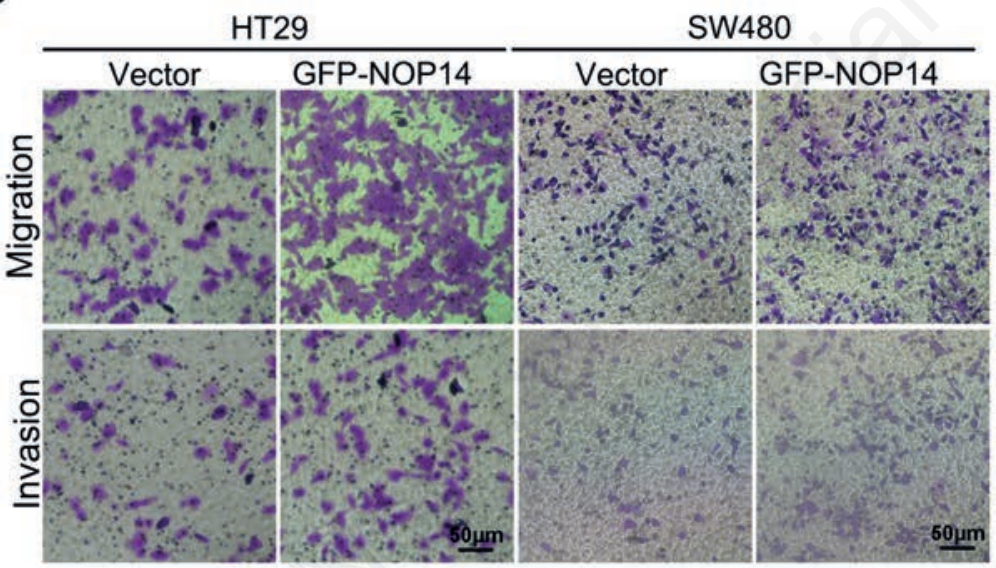

B

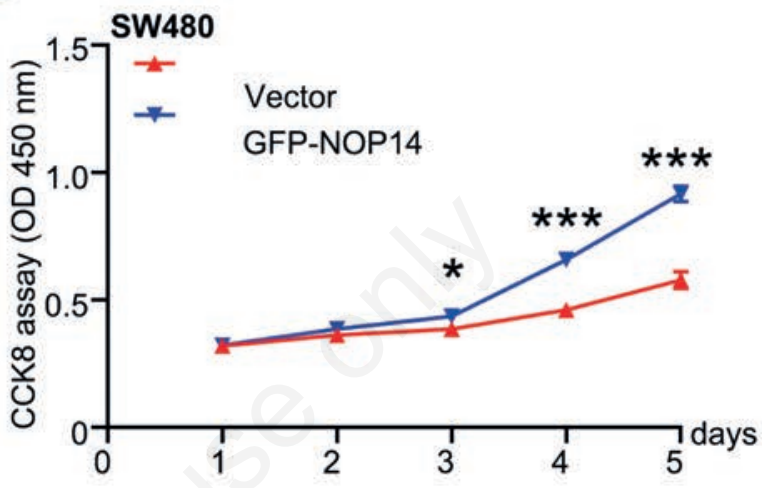

D

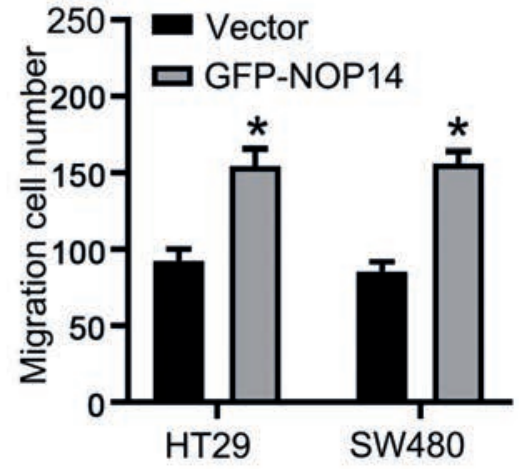

E

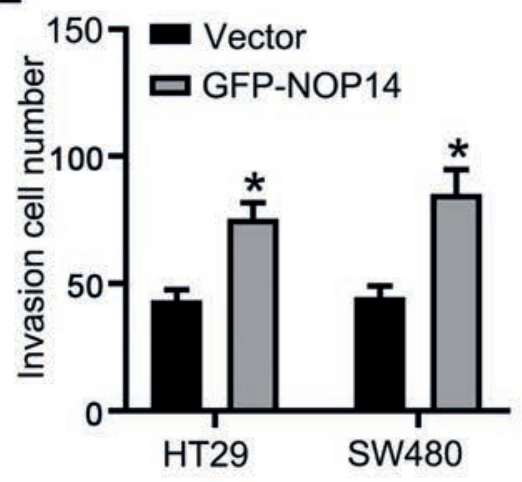

Figure 3. NOP14 effect on the proliferation of CRC, invasion, and migration overexpression. Cell growth was detected by the method of CCK8 vector and GFP-NOP14 cytotoxicity in cultured HT29 (A) and SW480 (B) cells; the migration and invasion assay were measured by transwell in HT29 and SW480 cells, presentative images were shown in C, and the data of migration (D) and invasion (E) were calculated. ${ }^{*} \mathrm{p}<0.05,{ }^{* *} \mathrm{p}<0.01$ compared with the Vector group. Scale bar: $20 \mu \mathrm{m}$. 
A

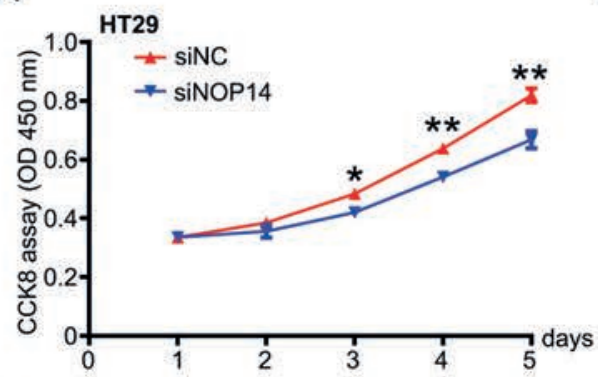

C
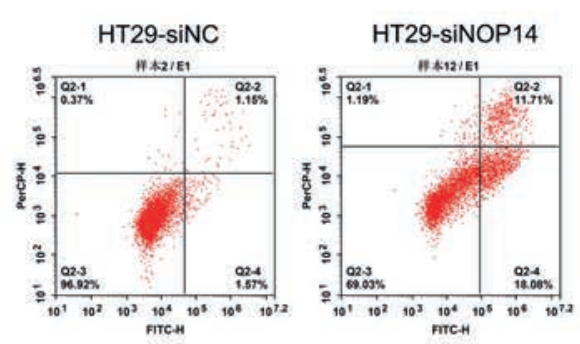

E
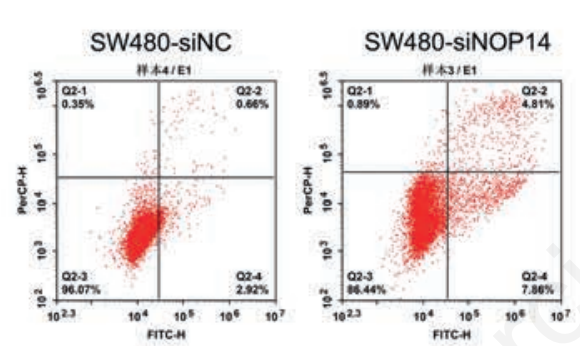

B

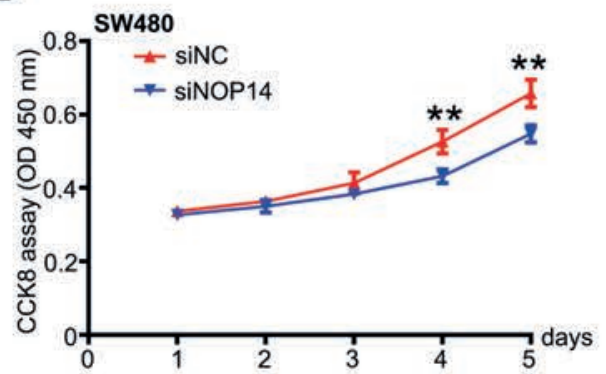

$\mathrm{D}$

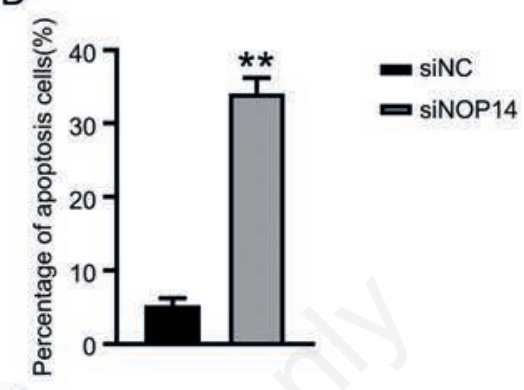

$\mathrm{F}$

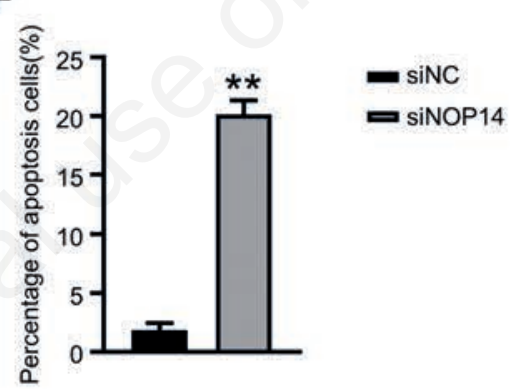

G
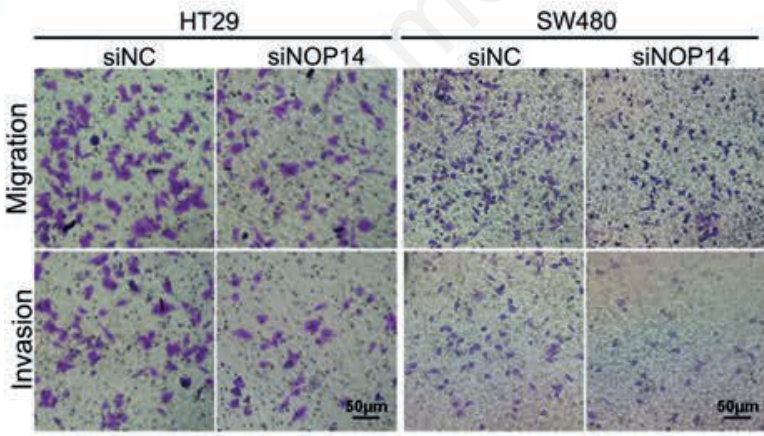

$\mathrm{H}$
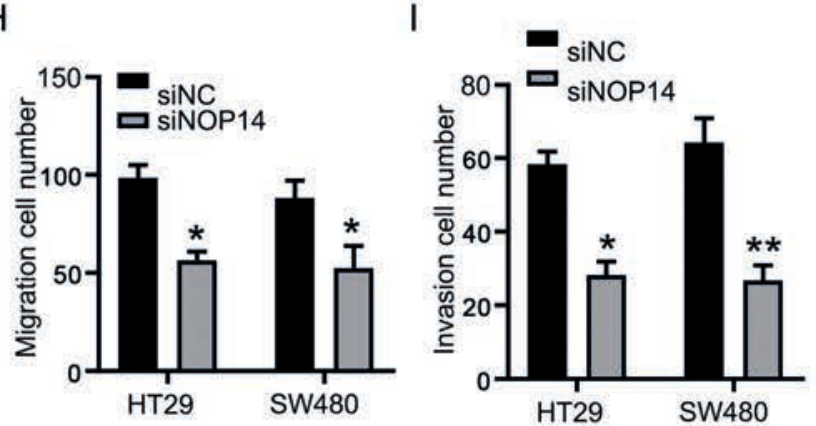

Figure 4. NOP14 of CRC knockdown on cell proliferation, apoptosis, invasion, and migration. A-B) Detect siNC and siNOP14 cytotoxicity in the methods and with HT29 cells cultured SW480 cells of CCK8. C-F) Used in HT29 cells and cultured SW480 cells transfected siNOP14 flow cytometry on CRC cells apoptosis. G-I) SW480 and HT29 cells in culture cells, and transfection siNC siNOP14, migration and invasion of cells detected using transwell method. ${ }^{*} \mathbf{p}<0.05 ;{ }^{* *} \mathrm{p}<0.01$. Scale bar: $50 \mu \mathrm{m}$. 
pension was aspirated at $2.5-5 \times 10^{5}$ cells per chamber and placed in a $37^{\circ} \mathrm{C}, 5 \% \mathrm{CO}_{2}$ incubator for $24-48 \mathrm{~h}$. Transwell system (Corning, Lowell, MA, USA) was applied. The upper chamber cells were wiped with a cotton swab, and the lower chamber surface cells fixed with $4 \%$ paraformaldehyde. Cells then stain with $0.1 \%$ crystal violet for $10 \mathrm{~min}$. After washing with water, images were taken under an optical microscope (IX81, Olympus, Tokyo, Japan) and the cells counted. For each group, counts from five high-power fields were randomly selected, and their average value calculated. For the cell invasion ability assays, six hours before cell inoculation, 10\% Matrigel glue (Corning BioCoat, Corning, NY, USA) was pre-laid inside the transwell chamber.

\section{In vivo tumorigenicity}

Four-week-old pathogen-free BALB/c mice were purchased from the Animal Center of Jinan University (Guangzhou, China). Five animals were in each shNOP14 and shNC group. All animals were housed at a temperature of $25 \pm 3^{\circ} \mathrm{C}$ and a relative humidity of $50 \pm 1 \%$ in a 12 -h light/dark cycle with free access to food and water. HT29 cells with NOP14 genetic knockdown, or with NC control, were inoculated into the armpit of the mice at $1 \times 10^{8}$ cells $/ \mathrm{mL}$. The tumor volumes were measured weekly and calculated using the following formula: $\pi / 6 \times$ length $\times$ width. ${ }^{2}$ Four weeks later, mice were sacrificed, and the tumors were weighed.

\section{Statistical analysis}

Statistical analysis was performed using SPSS 20.0 software (IBM, Armonk, NY, USA). Data were represented as mean \pm SD. For two group comparisons, Student's $t$-tests were used; for multiple group comparisons, one-way analysis of variance (ANOVA) was applied; p-values $<0.05$ were taken to indicate statistically significant differences.

\section{Results}

\section{NOP14 was upregulated in clinical samples of colorectal cancer}

To determine the gene expression and regulation mechanism of colon cancer development, we collected clinical tissue samples of colorectal cancers, with adjacent normal tissues as controls. Immunohistochemistry indicated that the expression level of NOP14 in CRC samples was higher than that in precancerous tissues (Figure 1A). Four pairs of samples were subjected to Western blotting, and the data showed that the protein levels of NOP14 in CRC tissues were significantly higher than those in benign paracancerous tissues (Figure $1 \mathrm{~B}, \mathrm{C}$ ). The fluorescence quantitative PCR (qPCR) assay revealed that the relative expression level of NOP14 mRNA in carcinoma was also significantly increased (Figure 1D). Taken together, these data indicate that NOP14 was upregulated in CRC tissues.

\section{NOP14 was upregulated in colorectal cancer cells}

To investigate the impact of NOP14 in CRC cells, we cultured normal colonic epithelial cells (NCM460) and colon cancer cells (Lovo, SW480, HCT116, HT29 and DLD1). Western blotting was performed to detect the expression level of NOP14 (Figure 2A). Increased NOP14 protein levels were found in cancer cells compared to normal cells (Figure 2B). The levels of NOP14 mRNA qualified using qPCR in colon cancer cells showed the same trend as protein levels (F Figure $2 \mathrm{C}$ ). These results indicate that NOP14 was also increased in CRC cells.

\section{NOP14 overexpression promoted cell proliferation,} invasion, and migration of colorectal cancer

Two CRC cell lines, HT29 and SW48, were cultured, to further investigate the impact of NOP14 on cancer development. NOP14 was overexpressed by transfecting cells with a GFP-NOP14 encoding plasmid, and the cell viability was detected by CCK8 assay. As shown in Figure $3 \mathrm{~A}$ and $\mathrm{B}, \mathrm{NOP} 14$ overexpression markedly promoted cell growth in these two colon cancer cell lines. Overexpression of NOP14 significantly promoted cell migration and invasion, as revealed by transwell assays (Figure 3C). Data related to cell migration and invasion are shown in Figure 3 $\mathrm{D}, \mathrm{E}$. Taken together, the data suggest that overexpression of NOP14 accelerated cell growth, invasion, and migration in CRC.
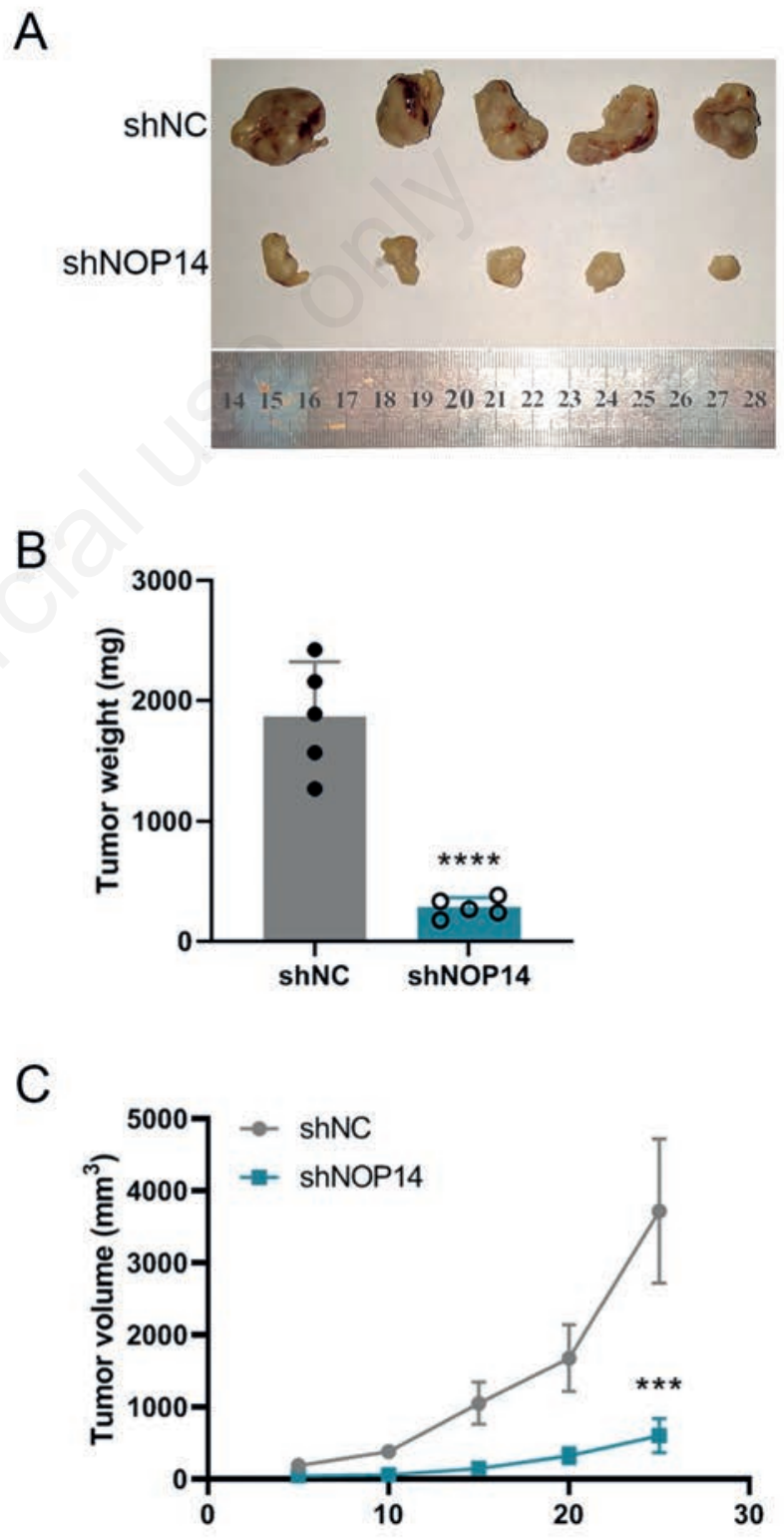

Figure 5. Silencing of NOP14 suppresses the tumorigenesis of CRC in vivo. A) Representative images of tumors from BALB/c mice 4 weeks after inoculation in shNOP14 and shNC HT29 CRC cells. B) The average tumor weight in the two groups. (C) The average tumor volume at each time point. ${ }^{* * *} \mathbf{p}<0.001 \mathrm{com}-$ pared with the shNC group. 
Silencing NOP14 promoted apoptosis and inhibited proliferation, invasion, and migration in colorectal cancer cells

NOP14 was genetically silenced in two colon cancer cell lines, HT29 and SW48, to determine its impact. The siRNA effects of the fragments were determined as previously reported. ${ }^{28}$ Transfection of the siRNA fragments against NOP14 significantly inhibited cell growth in these two cancer cell lines (Figure 4 A,B). Flow cytometry showed that the apoptosis rates in both HT29 and SW48 cell lines were significantly increased upon the introduction of NOP14 siRNA (Figure $4 \mathrm{C}, \mathrm{E}$ ). The quantified data are shown in Figure 4 D,F. Genetic knockdown of NOP14 inhibited cell migration and invasion in both cell lines (Figure 4G), and the quantified data showed the same results (Figure $4 \mathrm{H}, \mathrm{I}$ ). Taken together, these results indicate that NOP14 silencing induced apoptosis and inhibited cell proliferation, invasion, and migration in CRC.

\section{Inhibition of NOP14 suppressed tumorigenesis in colorectal cancer in vivo}

To investigate the role of NOP14 in tumorigenesis in vivo, HT29 cells that stably expressed NOP14-shRNA (shNOP14) or control-shRNA (shNC) were constructed using a lentivirus technique. Cells were inoculated into the armpit of BALB/c nude mice. Following inoculation, the tumor size was calculated each week, and mice were sacrificed and the tumors were collected four weeks after inoculation. As shown in Figure 5A, the tumors of the mice in the shNOP14 group were significantly smaller than those in the shNC group. The average tumor weight in shNOP14 group also decreased significantly over that in control mice (Figure 5B). The average tumor volume in the shNOP14 group grew more slowly than that of shNC group (Figure 5C). These results indicate that NOP14 promoted the progression of CRC in vivo, and inhibition of NOP14 decreased tumorigenesis in CRC.

\section{NOP14 functioned by modulating the Wnt/ $\beta$-catenin pathway}

To further explore the detailed mechanisms of NOP14 in the regulation of tumorigenesis in $\mathrm{CRC}$, we attempted to determine the involvement of NOP14 in the $\mathrm{Wnt} / \beta$-catenin pathway. As shown in Figure 6A, Western blotting assays were performed, and the results indicated that the levels of NRIP1, GSK-3 $\beta$ phosphorylation (but not total GSK-3 $\beta$ level), and $\beta$-catenin were elevated by overexpression of NOP14 encoding plasmids in CRC cells. Genetic knockdown of NOP14 significantly suppressed the aforementioned protein levels (Figure $6 \mathrm{~A}, \mathrm{~B}$ ). These results indicate that NOP14 modulated the Wnt/ $\beta$-catenin pathway.

\section{Discussion}

$\mathrm{CRC}$ is a severe malignant cancer of the digestive tract, with a incidence rate ranking third among all the cancers. ${ }^{1}$ Failure to detect CRC early may be one of the reasons for the poor prognosis in patients. Therefore, there is an urgent need for the identification of oncogenes. In this research, using immunohistochemistry, fluorescence quantitative PCR, and Western blotting, we detected the mRNA and protein levels of NOP14 in samples of benign and CRC tissues, and found that NOP14 mRNA and protein levels were significantly upregulated in cancer tissues. These data are consistent with the those in the literature pertaining to other cancers. ${ }^{29,31}$ Overexpression of NOP14 promoted the cell proliferation, migration, and invasion of colorectal cells, while genetic knockdown of NOP14 suppressed these behaviors, suggesting that NOP14 is sufficient and necessary for the development of colon cancer.

NOP14 is important in tumor progression, including cell proliferation, metastasis, and apoptosis. ${ }^{26,32-36}$ NOP14 is one of the direct targets of miR-502-5p in bladder cancer. ${ }^{29}$ NOP14 has been
A

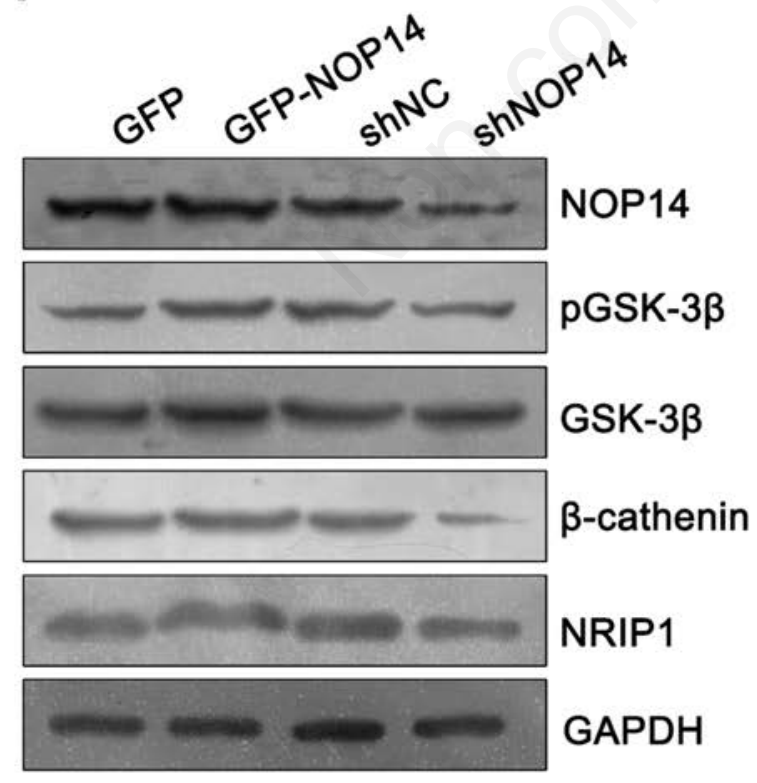

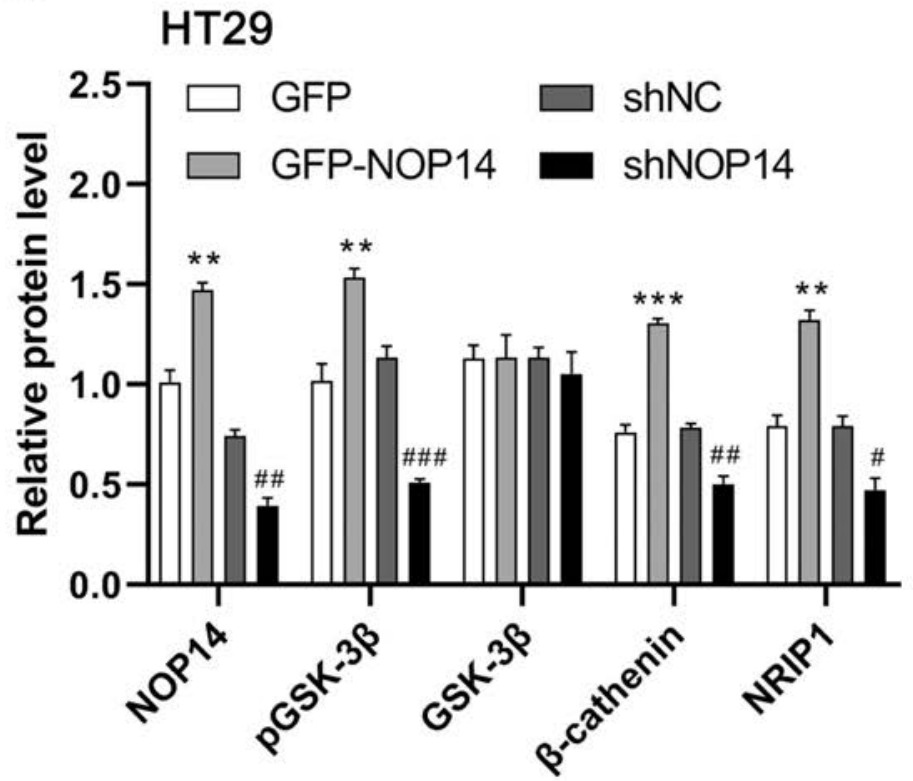

Figure 6. NOP14 modulate the NRIP1/GSK-3 $/ \beta$-catenin signalling pathway in HT29 cells. A) Cultured HT29 cells were transfected with NOP14 overexpression (GFP-NOP14) or knockdown (shNOP14) plasmids; cell lysates were collected for Western blotting analy-

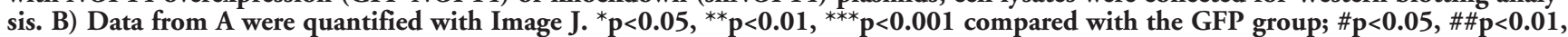
$\# \#$ p $<0.001$ compared with the shNC group. 
identified to be a specific target of Vioprolide A, indicating an involvement in ribosome biogenesis in human acute lymphoblastic leukemia. ${ }^{37}$ NOP14 suppresses breast cancer tumorigenesis and metastasis by enhancing ER $\alpha$ expression and inhibiting the Wnt pathway. ${ }^{38}$ NOP14 is overexpressed in pancreatic ductal adenocarcinoma (PDAC) cells and metastatic tissue samples. ${ }^{30}$ Overexpression of NOP14 promotes cell growth, whereas its suppression inhibits mobility of PDAC cells. NOP14 regulates the level of mutp53 mRNA and suppresses miR-174-5p induced p21 expression in PDAC cells. ${ }^{30}$ In pancreatic cancer, inhibition of NOP14 reduces, while upregulation of NOP14 promotes cell motility, growth and metastasis in vivo and in vitro. ${ }^{32}$ In lung and liver cancer cells with induced DNA damage, the lncRNA NOP14AS1 is upregulated, while the mRNA of NOP14 is downregulated, indicating the critical impact of the inverse regulation of NOP14AS1:NOP14 expression in a p53-dependent manner in DNA damage.$^{39}$ In the melanoma cell lines SK-MEL-110 and A-375, NOP14 is targeted by miR-122-5p to regulate cell proliferation, the cell cycle, and apoptosis. ${ }^{40}$ However, there is controversial evidence showing the NOP14 might be downregulated in the blood cells of ovarian cancer patients. ${ }^{41}$ In the current study, we fund that NOP14 was overexpressed in CRC cancer tissues and cells. Collectively, this evidence suggests that NOP14 plays an important role in the regulation of biochemical functions during cancer development.

The Wnt/GSK-3 $\beta / \beta$-catenin pathway has been reported to be involved in several cancers, including breast cancer, ${ }^{42}$ lung cancer, ${ }^{43}$ osteosarcoma ${ }^{44}$ and CRC. ${ }^{45}$ Abnormal accumulation of $\beta$ catenin is frequently reported in CRC. ${ }^{46}$ Uncontrolled activation of the Wnt/GSK-3 $\beta / \beta$-catenin pathway always leads to tumorigenesis. ${ }^{47}$ NRIP1 has been reported to be the downstream effector of NOP $14{ }^{48,49}$ In colon cancers, NRIP1 regulates intestinal homeostasis and tumorigenesis via upregulation of the expression of APC, which is a component of the $\mathrm{Wnt} / \mathrm{APC} / \beta$-catenin signaling pathway. ${ }^{50,51}$ Thus, NRIP1 is closely related to the GSK- $3 \beta / \beta$-catenin pathway. In the current study, NOP14 expression was closely related to NRIP1 level, indicating that NRIP1 might be an effector for the transduction of NOP14-regulated CRC development. NOP14 increased the level of phosphorylated GSK-3 $\beta$ downstream of $\beta$ catenin, indicating the NOP14 exerts its effects through the GSK$3 \beta / \beta$-catenin pathway, a finding which is consistent with reports in melanoma cancer. ${ }^{52}$ We suppose that NOP14 might regulate NRIP1 to modulate the function of GSK-3 $\beta / \beta$-catenin pathway. Further studies should be carried out to determine the relationship between GSK-3 $\beta / \beta$-catenin and NRIP1.

Taken together, this study further clarifies the processes involved in the development of CRC and provides evidence for the mechanism of NOP14 regulation. It suggests new approaches and potential targets for the clinical treatment of CRC.

\section{References}

1. Bray F, Ferlay J, Soerjomataram I, Siegel RL, Torre LA, Jemal A. Global cancer statistics 2018: GLOBOCAN estimates of incidence and mortality worldwide for 36 cancers in 185 countries. CA Cancer J Clin 2018;68:394-424.

2. Zhou Z, Mo S, Dai W, Xiang W, Han L, Li Q, et al. Prognostic nomograms for predicting cause-specific survival and overall survival of stage I-III colon cancer patients: a large populationbased study. Cancer Cell Int 2019;19:355.

3. Advani SM, Advani PS, Brown DW, DeSantis SM, Korphaisarn K, VonVille HM, et al. Global differences in the prevalence of the $\mathrm{CpG}$ island methylator phenotype of colorectal cancer. BMC Cancer 2019;19:964.

4. Zheng J, Park MH, Son DJ, Choi MG, Choi JS, Nam KT, et al.
(E)-4-(3-(3,5-dimethoxyphenyl)allyl)-2-methoxyphenol inhibits growth of colon tumors in mice. Oncotarget 2015;6:41929-43.

5. Cao G, Cheng D, Ye L, Pan Y, Yang F, Lyu S. Surgical resection of pulmonary metastases from colorectal cancer: 11 years of experiences. PLoS One 2017;12:e0175284.

6. Araghi M, Soerjomataram I, Jenkins M, Brierley J, Morris E, Bray F, et al. Global trends in colorectal cancer mortality: projections to the year 2035. Int J Cancer 2019;144:2992-3000.

7. Keum N, Giovannucci E. Global burden of colorectal cancer: emerging trends, risk factors and prevention strategies. Nat Rev Gastroenterol Hepatol 2019;16:713-32.

8. Dawson H, Kirsch R, Messenger D, Driman D. A review of current challenges in colorectal cancer reporting. Arch Pathol Lab Med 2019;143:869-82.

9. Saad AM, Abdel-Rahman O. Initial systemic chemotherapeutic and targeted therapy strategies for the treatment of colorectal cancer patients with liver metastases. Expert Opin Pharmacother 2019;20:1767-75.

10. Zhu DJ, Chen XW, OuYang MZ, Lu Y. Three surgical planes identified in laparoscopic complete mesocolic excision for right-sided colon cancer. World J Surg Oncol 2016;14:7.

11. Zhang Y, Chen Z, Li J. The current status of treatment for colorectal cancer in China: A systematic review. Medicine (Baltimore) 2017;96:e8242.

12. Gu MJ, Huang QC, Bao CZ, Li YJ, Li XQ, Ye D, et al. Attributable causes of colorectal cancer in China. BMC Cancer 2018;18:38.

13. Siegel R, DeSantis C, Virgo K, Stein K, Mariotto A, Smith T, et al. Cancer treatment and survivorship statistics, 2012. CA Cancer J Clin 2012;62:220-41.

14. Catalano I, Trusolino L. The Stromal and Immune Landscape of Colorectal Cancer Progression during Anti-EGFR Therapy. Cancer Cell 2019;36:1-3.

15. Kennedy SA, Jarboui MA, Srihari S, Raso C, Bryan K, Dernayka L, et al. Extensive rewiring of the EGFR network in colorectal cancer cells expressing transforming levels of KRAS(G13D). Nat Commun 2020;11:499.

16. Khan K, Valeri N, Dearman C, Rao S, Watkins D, Starling N, et al. Targeting EGFR pathway in metastatic colorectal cancertumour heterogeniety and convergent evolution. Crit Rev Oncol Hematol 2019;143:153-63.

17. Rahbari NN, Kedrin D, Incio J, Liu H, Ho WW, Nia HT, et al. Anti-VEGF therapy induces ECM remodeling and mechanical barriers to therapy in colorectal cancer liver metastases. Sci Transl Med 2016;8:360ra135.

18. Bhattacharya R, Ye XC, Wang R, Ling X, McManus M, Fan F, et al. Intracrine VEGF Signaling Mediates the Activity of Prosurvival Pathways in Human Colorectal Cancer Cells. Cancer Res 2016;76:3014-24.

19. Firestein R, Bass AJ, Kim SY, Dunn IF, Silver SJ, Guney I, et al. CDK8 is a colorectal cancer oncogene that regulates betacatenin activity. Nature 2008;455:547-51.

20. Chatel G, Ganeff C, Boussif N, Delacroix L, Briquet A, Nolens $\mathrm{G}$, et al. Hedgehog signaling pathway is inactive in colorectal cancer cell lines. Int J Cancer 2007;121:2622-7.

21. Qiao L, Wong BC. Role of Notch signaling in colorectal cancer. Carcinogenesis 2009;30:1979-86.

22. Matsushita M, Matsuzaki K, Date M, Watanabe T, Shibano K, Nakagawa T, et al. Down-regulation of TGF-beta receptors in human colorectal cancer: implications for cancer development. Br J Cancer 1999;80:194-205.

23. Slattery ML, Lundgreen A, Kadlubar SA, Bondurant KL, Wolff RK. JAK/STAT/SOCS-signaling pathway and colon and rectal cancer. Mol Carcinog 2013;52:155-66. 
24. Wach A, Kałuzińska K, Frączek P. PI3K-Akt and Ras-RafMAPK signaling in colorectal cancer - Comparison of activity in primary tumor tissues and primary tumour - Derived human colorectal cancer cell lines: PS122. Porto Biomed J 2017;2:215-6.

25. Milkereit P, Strauss D, Bassler J, Gadal O, Kühn H, Schütz S, et al. A Noc complex specifically involved in the formation and nuclear export of ribosomal $40 \mathrm{~S}$ subunits. J Biol Chem 2003;278:4072-81.

26. Kühn H, Hierlmeier T, Merl J, Jakob S, Aguissa-Touré AH, Milkereit $\mathrm{P}$, et al. The Noc-domain containing C-terminus of Noc $4 p$ mediates both formation of the Noc4p-Nop14p submodule and its incorporation into the SSU processome. PLoS One 2009; 4:e8370.

27. Hannes F, Hammond P, Quarrell O, Fryns JP, Devriendt K, Vermeesch JR. A microdeletion proximal of the critical deletion region is associated with mild Wolf-Hirschhorn syndrome. Am J Med Genet A 2012;158a:996-1004.

28. Li J, Fang R, Wang J, Deng L. NOP14 inhibits melanoma proliferation and metastasis by regulating $\mathrm{Wnt} / \beta$-catenin signaling pathway. Braz J Med Biol Res 2018;52:e7952.

29. Ying Y, Li J, Xie H, Yan H, Jin K, He L, et al. CCND1, NOP14 and DNMT3B are involved in miR-502-5p-mediated inhibition of cell migration and proliferation in bladder cancer. Cell Prolif 2020;53:e12751.

30. Du Y, Liu Z, You L, Hou P, Ren X, Jiao T, et al. Pancreatic Cancer Progression Relies upon Mutant p53-Induced Oncogenic Signaling Mediated by NOP14. Cancer Res 2017;77:2661-73.

31. Chang SY, Huang J, Niu H, Wang J, Si Y, Bai ZG, et al. Epigenetic regulation of osteopontin splicing isoform $\mathrm{c}$ defines its role as a microenvironmental factor to promote the survival of colon cancer cells from 5-FU treatment. Cancer Cell Int. 2020;14;20:452.

32. Zhou B, Wu Q, Chen G, Zhang TP, Zhao YP. NOP14 promotes proliferation and metastasis of pancreatic cancer cells. Cancer Lett 2012;322:195-203.

33. Liu PC, Thiele DJ. Novel stress-responsive genes EMG1 and NOP14 encode conserved, interacting proteins required for 40S ribosome biogenesis. Mol Biol Cell 2001;12:3644-57.

34. Zhou B, Irwanto A, Guo YM, Bei JX, Wu Q, Chen G, et al. Exome sequencing and digital PCR analyses reveal novel mutated genes related to the metastasis of pancreatic ductal adenocarcinoma. Cancer Biol Ther 2012;13:871-9.

35. Cao Q, Wang X, Zhao M, Yang R, Malik R, Qiao Y, et al. The central role of EED in the orchestration of polycomb group complexes. Nat Commun 2014;5:3127.

36. Woods NT, Mesquita RD, Sweet M, Carvalho MA, Li X, Liu $\mathrm{Y}$, et al. Charting the landscape of tandem BRCT domainmediated protein interactions. Sci Signal 2012;5:rs6.

37. Kirsch VC, Orgler C, Braig S, Jeremias I, Auerbach D, Müller $\mathrm{R}$, et al. The cytotoxic natural product vioprolide A targets nucleolar protein 14, which is essential for ribosome biogenesis. Angew Chem Int Ed Engl 2020;59:1595-600.

38. Lei JJ, Peng RJ, Kuang BH, Yuan ZY, Qin T, Liu WS, et al. NOP14 suppresses breast cancer progression by inhibiting
NRIP1/Wnt/ק-catenin pathway. Oncotarget 2015;6:25701-14.

39. Goyal A, Fiskin E, Gutschner T, Polycarpou-Schwarz M, Gross M, Neugebauer J, et al. A cautionary tale of sense-antisense gene pairs: independent regulation despite inverse correlation of expression. Nucleic Acids Res 2017;45:12496-508.

40. Li J, Zhao R, Fang R, Wang J. [miR-122-5p inhibits the proliferation of melanoma cells by targeting NOP14].[Article in Chinese]. Nan Fang Yi Ke Da Xue Xue Bao 2018;38:1360-5.

41. Isaksson HS, Sorbe B, Nilsson TK. Whole genome expression profiling of blood cells in ovarian cancer patients-prognostic impact of the CYP1B1, MTSS1, NCALD, and NOP14. Oncotarget 2014;5:4040-9.

42. Wang H, Sun L, Jiang J, Yu S, Zhou Q. Suppression of the proliferation and invasion of breast cancer cells by ST7L occurs through inhibition of activation of Wnt/GSK-3beta/betacatenin signalling. Clin Exp Pharmacol Physiol 2020;47:11926.

43. Liu C, Liu L, Zhang Y, Jing H. Molecular mechanism of aquapontin (AQP3) in regulating differentiation and apoptosis of lung cancer stem cells through Wnt/GSK-3beta/betaCatenin pathway. J BUON 2020;25:828-34.

44. Lv YF, Dai H, Yan GN, Meng G, Zhang X, Guo QN. Downregulation of tumor suppressing STF cDNA 3 promotes epithelial-mesenchymal transition and tumor metastasis of osteosarcoma by the Wnt/GSK-3beta/beta-catenin/Snail signaling pathway. Cancer Lett 2016;373:164-73.

45. Lv S, Zhang J, He Y, Liu Q, Wang Z, Liu B, et al. MicroRNA520e targets AEG-1 to suppress the proliferation and invasion of colorectal cancer cells through Wnt/GSK-3beta/beta-catenin signalling. Clin Exp Pharmacol Physiol 2020;47:158-67.

46. White BD, Chien AJ, Dawson DW. Dysregulation of Wnt/beta-catenin signaling in gastrointestinal cancers. Gastroenterology 2012;142:219-32.

47. Polakis P. Wnt signaling in cancer. Cold Spring Harb Perspect Biol 2012;4: a008052.

48. Zhou D, Quach KM, Yang C, Lee SY, Pohajdak B, Chen S. PNRC: a proline-rich nuclear receptor coregulatory protein that modulates transcriptional activation of multiple nuclear receptors including orphan receptors SF1 (steroidogenic factor 1) and ERRalpha1 (estrogen related receptor alpha-1). Mol Endocrinol 2000;14:986-98.

49. Cavailles V, Dauvois S, L'Horset F, Lopez G, Hoare S, Kushner PJ, et al. Nuclear factor RIP140 modulates transcriptional activation by the estrogen receptor. EMBO J 1995; 14:3741-51.

50. Lei JJ, Peng RJ, Kuang BH, Yuan ZY, Qin T, Liu WS, et al. NOP14 suppresses breast cancer progression by inhibiting NRIP1/Wnt/beta-catenin pathway. Oncotarget 2015;6:2570114.

51. Lapierre M, Bonnet S, Bascoul-Mollevi C, Ait-Arsa I, Jalaguier S, Del Rio M, et al. RIP140 increases APC expression and controls intestinal homeostasis and tumorigenesis. J Clin Invest 2014;124:1899-913.

52. Li J, Fang R, Wang J, Deng L. NOP14 inhibits melanoma proliferation and metastasis by regulating Wnt/beta-catenin signaling pathway. Braz J Med Biol Res 2018;52:e7952.

Received for publication: 15 March 2021. Accepted for publication: 25 May 2021.

This work is licensed under a Creative Commons Attribution-NonCommercial 4.0 International License (CC BY-NC 4.0).

(C) Copyright: the Author(s), 2021

Licensee PAGEPress, Italy

European Journal of Histochemistry 2021; 65:3246

doi:10.4081/ejh.2021.3246 